Research Article

\title{
Application of a Stress-Fractional Model for Capturing the Stress-Strain Behavior of Granular Soils
}

\author{
Wei Lu $\left(\mathbb{D},{ }^{1}\right.$ Jiangong Yang $\left(\mathbb{D},{ }^{1}\right.$ and Jinan Wang $(\mathbb{D})^{2}$ \\ ${ }^{1}$ Tianjin College, University of Science and Technology Beijing, Tianjin 301800, China \\ ${ }^{2}$ School of Civil and Resource Engineering, University of Science and Technology Beijing, Beijing 100083, China \\ Correspondence should be addressed to Jiangong Yang; jgy_tju@163.com
}

Received 29 June 2021; Revised 13 August 2021; Accepted 7 September 2021; Published 22 September 2021

Academic Editor: Hadi Hasanzadehshooiili

Copyright $\odot 2021$ Wei Lu et al. This is an open access article distributed under the Creative Commons Attribution License, which permits unrestricted use, distribution, and reproduction in any medium, provided the original work is properly cited.

Granular soils usually serve as the filler material in geotechnical engineering. This study presents the development and application of a stress-fractional model for granular soils with different initial material states. To capture the plastic loading and flow behaviors, a subloading surface with the fractional stress gradient is used. The developed model contains twelve parameters which can be determined through triaxial tests. To validate the developed model, the well-documented test results of Firoozkuh No. 161 sand and crushed basalt are simulated and discussed. It is found that the stress-fractional model can reasonably simulate the undrained and drained behaviors of granular soils consolidated with different densities and mean effective pressures.

\section{Introduction}

Granular soils, e.g., ballast and sand, are often encountered or used in engineering practice. For example, ballast usually serves as an essential track construction layer to bear the repeated moving load transmitted by the train. During the whole operation period, a rail track usually experiences a large number of repeated train loads. Sand can be used as a filter material. Accurate prediction of the corresponding maintenance periods necessitates the development of an advanced constitutive model that captures ballast or sand deformation [1]. Although traditional elastoplastic constitutive models have been investigated widely and successfully applied in many fields, more effort is required to realistically describe the stress-strain relationship of granular soil, which was observed to be state-dependent and nonassociated. In classical plasticity theory, the dependence of associative or nonassociative plastic flow of granular soil on the material state can be mathematically elaborated by using a loading/ bounding/reference surface and a plastic potential surface with frictional parameters dependent on the material state. For example, a series of elegant constitutive models have been developed for predicting the instability and flow liquefaction behavior of Firoozkuh sand [2] as well as other sands.

However, different from the modelling techniques often used in traditional plasticity, a new modelling framework based on the fractional plasticity (FP) has been developed and implemented recently [3-6], in which the plastic potential was not necessarily used. However, the previous FP approach was established based on granular soil and the soil-structure interface [7], using the loading/ yielding surface. This study attempts to develop a statedependent constitutive model for granular soil by using the fractional plasticity and subloading surface concepts [8]. For simplicity, conventional stress and strain notations in soil mechanics, with compression positive and extension negative, are used. In addition, this study is limited to homogenous materials under isotropic or anisotropic consolidation.

\section{Constitutive Model}

In elastoplastic theory $[3,9]$, the tensorial incremental plastic strain, $\varepsilon_{i j}^{p}$, shall be obtained by 


$$
\begin{aligned}
\varepsilon_{i j}^{p} & =L n_{i j}, \\
L & =\frac{1}{K_{p}} m_{k l} \sigma_{k l}^{\prime},
\end{aligned}
$$

where $m_{k l}, n_{i j}$, and $K_{p}$ are the plastic loading tensor, plastic flow tensor, and plastic modulus, respectively, and $i, j, k$, and $l$ are indices running from 1 to 3 . is the Macaulay bracket. $\sigma_{k l}^{\prime}$ is the tensorial incremental effective stress. In this study, $m_{k l}$ defines a unit normal at the subloading surface $(f)$, and $n_{i j}$ defines the plastic strain rate direction. For the purpose of obtaining the analytical solution, Sun et al. [9] suggested to use the following fractional derivatives for modelling:

$$
\begin{aligned}
& { }_{a} D_{\sigma}^{\alpha} f\left(\sigma^{\prime}\right)=\frac{1}{\Gamma(n-\alpha)} \int_{a}^{\sigma^{\prime}} \frac{f^{(n)}(\chi) \mathrm{d} \chi}{(\sigma \prime-\chi)}, \quad \sigma^{\prime}>a, \\
& { }_{\sigma}^{\prime} D_{\sigma}^{\alpha} f\left(\sigma^{\prime}\right)=\frac{(-1)^{n}}{\Gamma(n-\alpha)} \int_{\sigma^{\prime}}^{a} \frac{f^{(n)}(\chi) \mathrm{d} \chi}{\left(\chi-\sigma^{\prime}\right)}, \quad \sigma^{\prime}>a \text {, }
\end{aligned}
$$

where the fractional order, $\alpha$, ranges between $n$ and $n-1$, with the integer $n>0$ [3]. $D$ denotes partial differentiation. $a$ stands for the integral limit in equations (3a) and (3b). Depending on the relative position of $a$ with regard to $\sigma l$, one should decide whether equation (3a) or (3b) should be used. Then, $m_{k l}$ and $n_{i j}$ can be expressed as follows [10]:

$$
\begin{aligned}
& m_{k l}=\frac{1}{\sqrt{1+d_{f}^{2}}}\left[\frac{d_{f}}{3} \delta_{k l}+\frac{3 s_{k l}}{2 q}\right], \\
& n_{i j}=\frac{1}{\sqrt{1+d_{g}^{2}}}\left[\frac{d_{g}}{3} \delta_{i j}+\frac{3 s_{i j}}{2 q}\right],
\end{aligned}
$$

where $\delta_{i j}$ is the Kronecker delta; the tensorial deviatoric stress, $s_{i j}$, can be defined as $s_{i j}=\sigma_{i j}^{\prime}-p^{\prime} \delta_{i j}$; the mean effective stress $\left(p^{\prime}\right)$ can be defined as $p^{\prime}=\sigma_{i j}^{\prime} \delta_{i j} / 3 . q=\sqrt{3 s_{i j} s_{i j} / 2}$ is the deviatoric stress. Although $m_{k l}$ and $n_{i j}$ incorporate the contributions from the deviatoric stress components, $\left(3 s_{k l} /(2 q)\right.$ or $\left.3 s_{i j} /(2 q)\right)$ and the volumetric parts $\left(d_{f} \delta_{k l} / 3\right.$ or $\left.d_{g} \delta_{i j} / 3\right)$, equations (4) and (5) assume a Drucker-Prager locus in the $\pi$-plane. $d_{f}$ and $d_{g}$ can be defined as

$$
\begin{gathered}
d_{f}=\frac{\partial f / \partial p^{\prime}}{\partial f / \partial q}, \\
d_{g}=\frac{\partial^{\alpha} f / \partial p^{\prime \alpha}}{\partial^{\alpha} f / \partial q^{\alpha} / \partial q^{\alpha}} .
\end{gathered}
$$

In addition, using the consistency condition and $H=L \widehat{H}, K_{p}$ can be determined as

$$
K_{p}=-\frac{\partial f}{\partial H} \widehat{H}
$$

where $\hat{H}$ is the law for isotropic hardening. Combining equations (1), (4), (5), and (8), the details of FP have now been provided.
In this study, the modified subloading surface [11] is used:

$$
f=M^{2} p^{\prime 2}+q^{2}-M^{2} p^{\prime 1+c} p_{0}^{\prime 1-c}=0,
$$

where $c$ is the material constant. $M$ is the slope of the critical state line (CSL) in the $p^{\prime}-q$ plane. $M=M_{c}$ for compression, and $M=M_{e}$ for extension. $p_{0}^{\prime}$ defines the location of the subloading surface. Combining equation (9) with equations (6) and (7), one has

$$
\begin{aligned}
& d_{f}=\frac{(1-c)_{M}^{2}-(1+c) \eta^{2}}{2 \eta} \\
& d_{g}=M^{1+\alpha} \frac{\left(p^{\prime}-p_{c}^{\prime}\right)+(2-\alpha)\left(p_{c}^{\prime}-p_{0}^{\prime} / 2\right)}{\left(q-q_{c}\right)+(2-\alpha) q_{c}},
\end{aligned}
$$

where $p_{c}^{\prime}=p_{a}\left[\left(e_{\Gamma}-e\right) / \lambda\right]^{1 / \xi}$ is the stress at the critical state; $p_{\mathrm{a}}=100 \mathrm{kPa}$ is the atmospheric pressure; $c=0$ is used when developing equation (11). $e_{\Gamma}, \lambda$, and $\xi$ are material constants describing the CSL in the $e-p^{\prime}$ plane. $e$ is the current void ratio.

$$
q_{c}=q+t M\left(p^{\prime}-p_{c}^{\prime}\right)
$$

where $t=+1$ for loading and $t=-1$ for unloading. The plastic modulus can be obtained as

$$
K_{p}=h_{0} G \frac{M_{p}-\eta}{\left|\eta-\eta_{\text {in }}\right|},
$$

where $h_{0}=\left(h_{1}-h_{2} e\right)(1+e) \exp (\beta \psi)$, in which $\beta, h_{1}$, and $h_{2}$ are material constants, and $\psi=e-e_{\Gamma}+\lambda\left(p_{c}^{\prime} / p_{a}\right)^{\xi} ; \eta_{\text {in }}$ is the stress ratio at the last stress reversal; $M_{p}=M \exp (-\beta \psi)$ is the stress ratio obtained at the peak state of granular soil under shearing. Moreover, incremental elastic strain $\left(\varepsilon_{i j}^{e}\right)$ is considered using Hooke's law:

$$
\varepsilon_{i j}^{e}=C_{i j k l}^{e} \sigma_{k l}^{\prime}
$$

with $C_{i j k l}^{e}=\left[-v \delta_{i j} \delta_{k l}+(1+\nu) / 2\left(\delta_{i k} \delta_{j l}+\delta_{i l} \delta_{j k}\right)\right] /[2 G \quad(1+$ $\nu)$ ] being the tensorial elastic compliance and $v$ being Poisson's ratio. $G=G_{0} F(e) \sqrt{p^{\prime} p_{a}}$ [12], where $F(e)=$ $(2.97-e)^{2} /(1+e)$ and $G_{0}$ is a material constant. It is noted that methods on how to determine the model constants from laboratory tests can be found in Sun et al. [3]. The values of the model constants for simulating railroad ballast and sand are shown in Table 1.

\section{Numerical Implementation}

In this section, the numerical methodology of the proposed FP model is given. Strain-controlled loading and the subsequent stress evolution are used in the numerical scheme, where there are five main procedures:

(1) For step $j(=1,2,3, \ldots, n)$, input the stress and strain conditions $\left(p^{\prime j}, q^{j}, \eta^{j}, e^{j}\right.$, and $\left.\psi^{j}\right)$ and calculate the $j$ th plastic loading and flow directions by using equations (4) and (5). 
TABLE 1: Model parameters.

\begin{tabular}{lcccccccccccc}
\hline Item & $e_{\Gamma}$ & $\lambda$ & $\xi$ & $M_{c}$ & $M_{e}$ & $\beta$ & $\alpha$ & $c$ & $h_{1}$ & $h_{2}$ & $G_{0}$ & $v$ \\
\hline Ballast & 1 & 0.129 & 0.6 & 1.9 & 1.3 & 2.1 & 1.02 & 0 & 1.8 & 1.8 & 73 & 0.25 \\
Sand & 0.923 & 0.057 & 0.5 & 1.22 & 0.87 & 4.0 & 1.02 & 0.75 & 3.5 & 3.5 & 100 & 0.11 \\
\hline
\end{tabular}

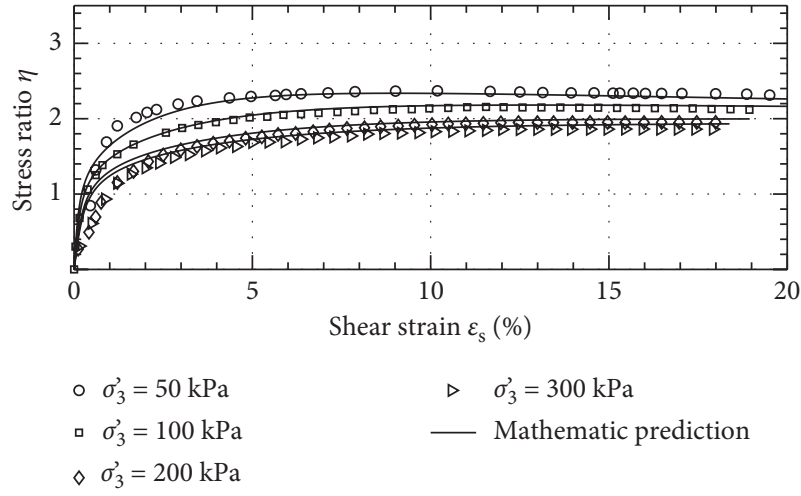

(a)

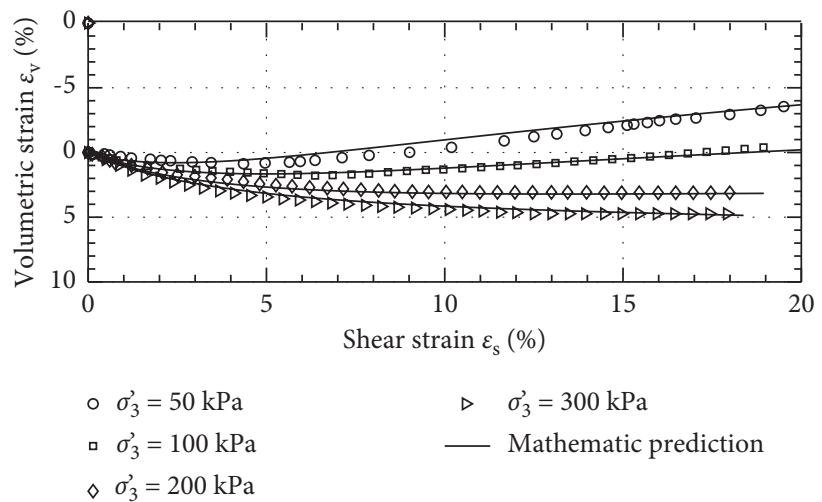

(b)

Figure 1: Observed and predicted monotonic behavior of crushed basalt [14].

(2) Then, calculate the hardening modulus $\left(H^{j}\right)$ as well as the elastoplastic compliance matrix $\left(\mathbf{C}^{j}\right)$ at step $j$ by using equations (1) and (11).

(3) Furthermore, compute the strain increments induced by axial loading $\left(\varepsilon^{j}\right)$ under drained loading.

(4) The induced stress increments $\left(\sigma^{\prime j}\right)$ can then be back-calculated using equation (14).

(5) Update the $j$ th total stress and strain matrices after obtaining the strain and stress increments by using the following:

$$
\begin{gathered}
\mathcal{\varepsilon}^{j+1}=\varepsilon^{j}+\varepsilon^{j}, \\
\sigma^{j+1}=\sigma^{j}+\sigma^{j} .
\end{gathered}
$$

Loading and unloading are judged by the dot product of the loading and incremental stress tensors, i.e., $m_{i j} \sigma_{i j}^{\prime} \geq 0$ for loading and $m_{i j} \sigma_{i j}^{\prime}<0$ for unloading. For more details, one can refer to Sun et al. [13]. Subroutines using the above methodology are then implemented in MATLAB for model simulation.

\section{Model Application}

This section provides the model application in simulating the real stress-strain behavior of ballast and sand subjected to element tests. The well-documented test data of crushed basalt [14] and Firoozkuh sand [2] are simulated and compared.

4.1. Ballast. Figure 1 shows the simulation results for the monotonic behavior of ballast with different initial material states [14]. All the laboratory tests were carried out on the saturated crushed basalt with a specimen size of $300 \mathrm{~mm}$ in diameter and $600 \mathrm{~mm}$ in height. For more details on test materials and test setup, please refer to Salim and Indraratna [14]. It is observed from Figure 1 that the proposed mathematical approach can well capture the stress-strain response of ballast, especially the stress dilation and strain hardening/softening induced by different material states.

Figures 2 and 3 provide the model simulations of the cyclic behavior of ballast with different initial states. In Figure 2, an initial void ratio $\left(e_{0}\right)$ of 0.65 and a confining pressure $\left(\sigma_{3}^{\prime}\right)$ of $50 \mathrm{kPa}$ are used for simulation. It can be observed that the shearing strain amplitude $\left(\delta \varepsilon_{1}\right)$ influences the cyclic behavior of ballast. If a small strain amplitude with higher frequency was applied, ballast would exhibit much more volumetric contraction and arrive at a higher strength more quickly, which indicates that a proper previbration with small strain amplitude and higher frequency can be a benefit for accelerating the ballast settlement.

In Figure $3, e_{0}$ of 0.65 and $\delta \varepsilon_{1}$ of $6 \%$ are used for simulation. It can be observed that the initial confining pressure has a significant influence on the cyclic behavior of ballast. If a weak confinement (lower $\sigma_{3}^{\prime}$ ) was applied, the material would exhibit much higher volumetric dilation and lower strength, which indicates the instability of the ballast layer. However, if a stronger confinement was applied, higher strength and enhanced densification of ballast can be achieved.

4.2. Sand. Figures 4-6 show the model simulations of the undrained behavior of Firoozkuh No. 161 sand with different initial material states provided by Lashkari et al. [2]. The Firoozkuh No. 161 sand was reported to have a coefficient of uniformity of 1.9 and a mean particle size of 0.24 . Test samples were $5 \mathrm{~cm}$ in diameter and $10 \mathrm{~cm}$ in height. Figure 4 


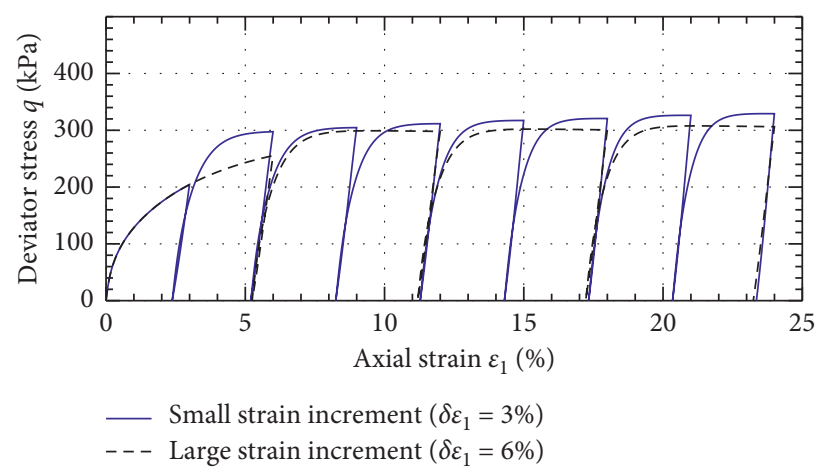

(a)

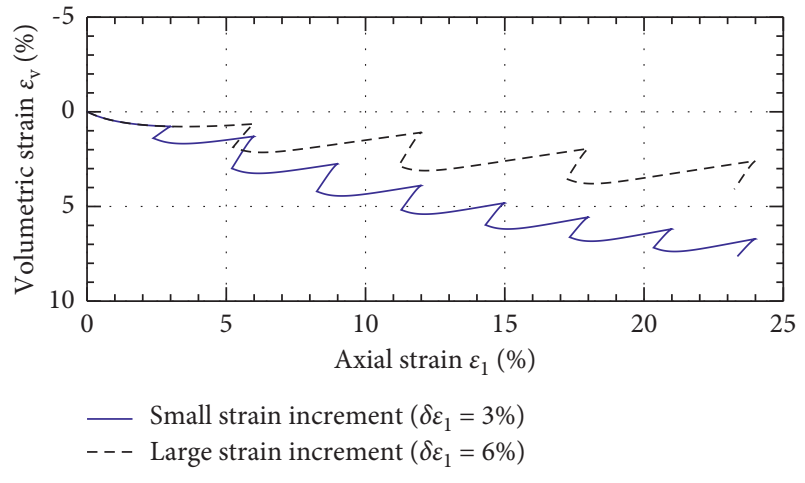

(b)

Figure 2: Predicted cyclic behavior of ballast under different strain increments.

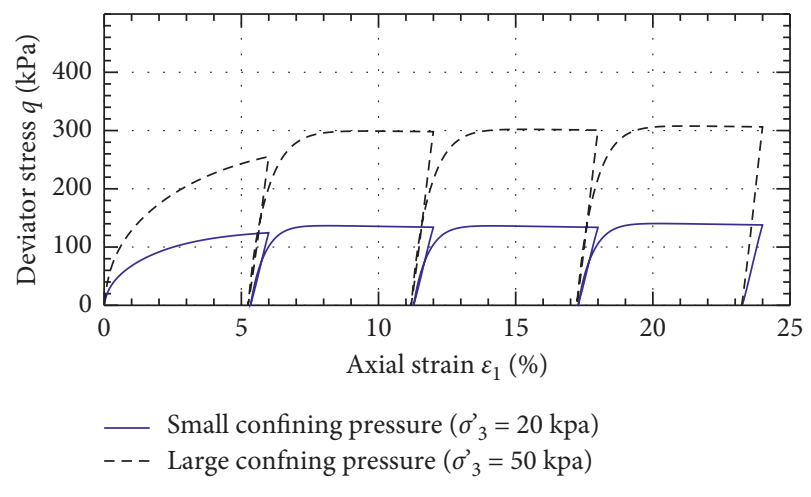

(a)

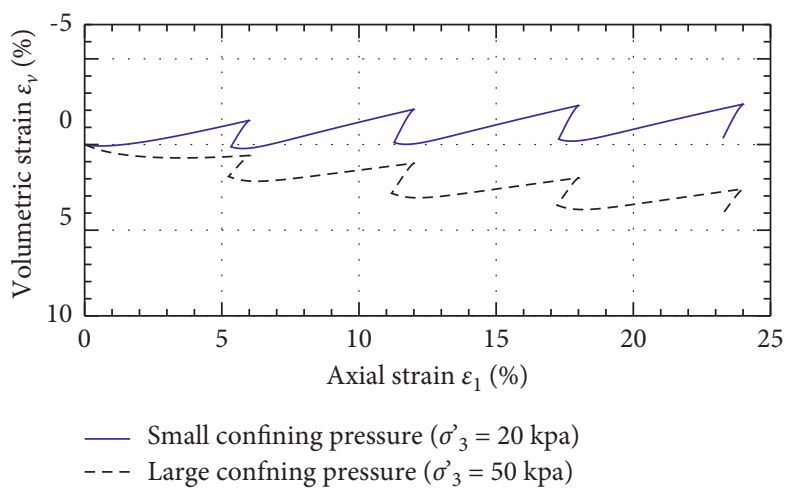

(b)

Figure 3: Predicted cyclic behavior of ballast under different initial pressures.

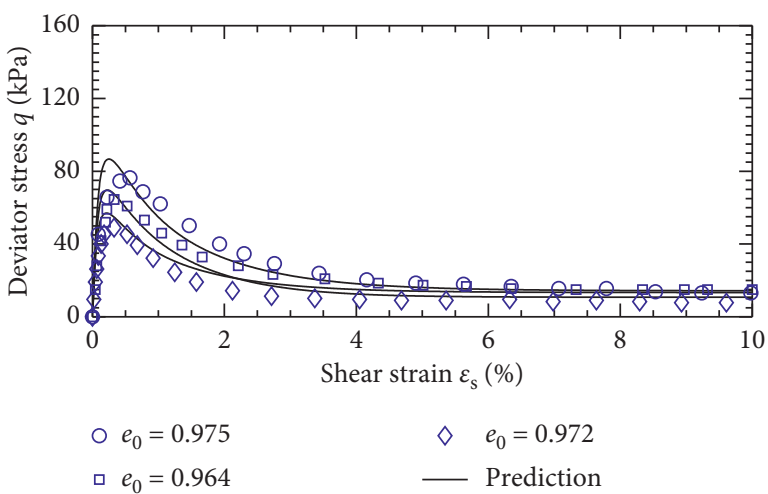

(a)

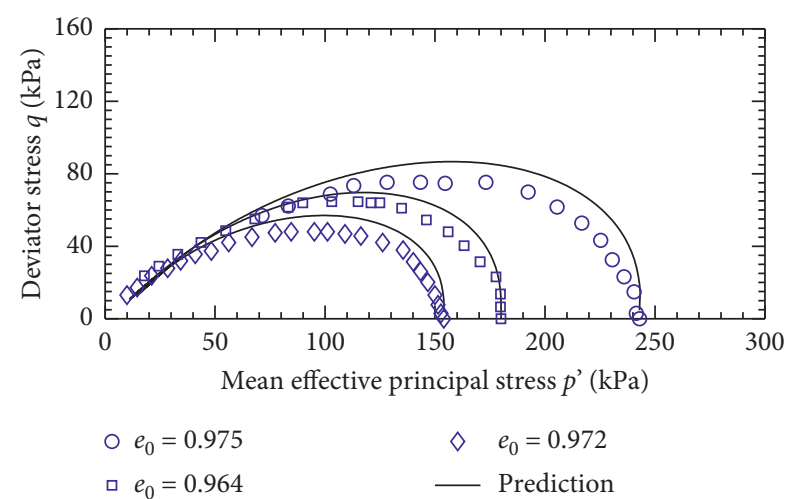

(b)

FIgURE 4: Simulations of the undrained behavior of loose Firoozkuh sand.

shows the model simulations of the loose Firoozkuh No. 161 sand under different initial confining pressures, where it can be found that the stress-fractional model can capture the undrained liquefaction behavior of loose Firoozkuh No. 161 sand. The effective stress paths are reasonably simulated. Figure 5 shows the model simulations of the undrained behavior of medium-dense Firoozkuh No. 161 sand, where a good agreement between the model simulations and the test results is observed. The predicted effective stresses first arrived at the phase transformation states and then mobilised towards the critical state. In addition to the test results under isotropic consolidation, Figure 6 shows the model simulation of the Firoozkuh No. 161 sand subjected to initially anisotropic consolidation. It can be found that the liquefaction and partial liquefaction behaviors of Firoozkuh No. 161 sand are well simulated. 


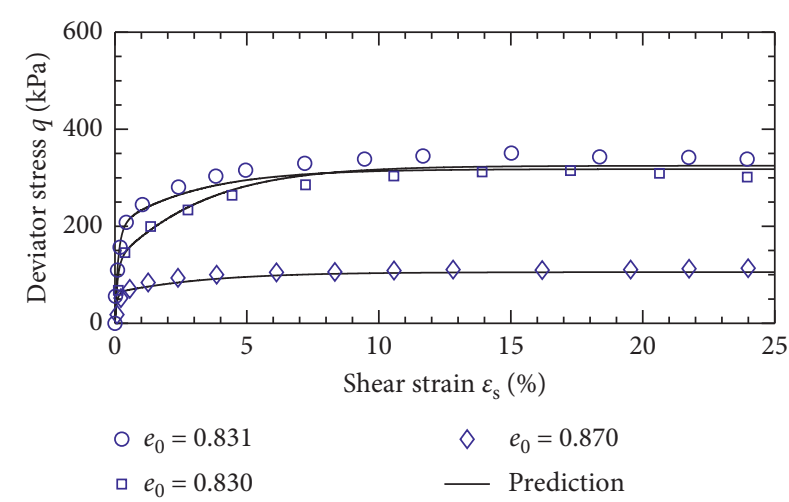

(a)

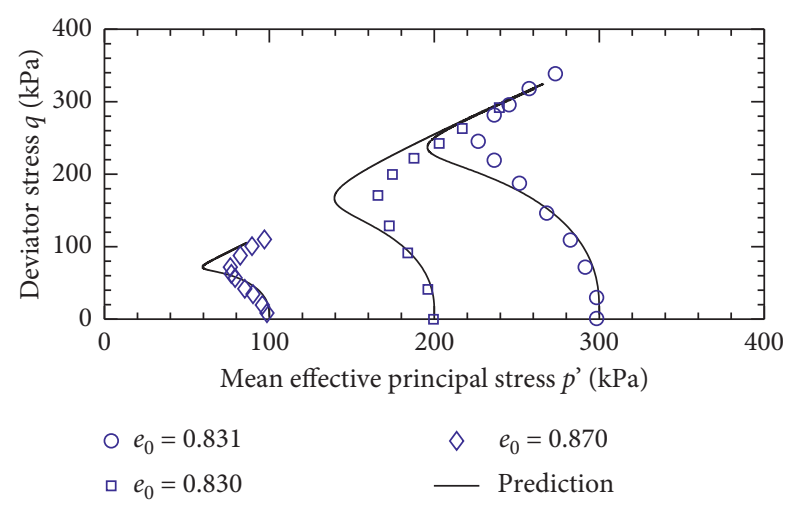

(b)

FIGURE 5: Simulations of the undrained behavior of medium-dense Firoozkuh sand.

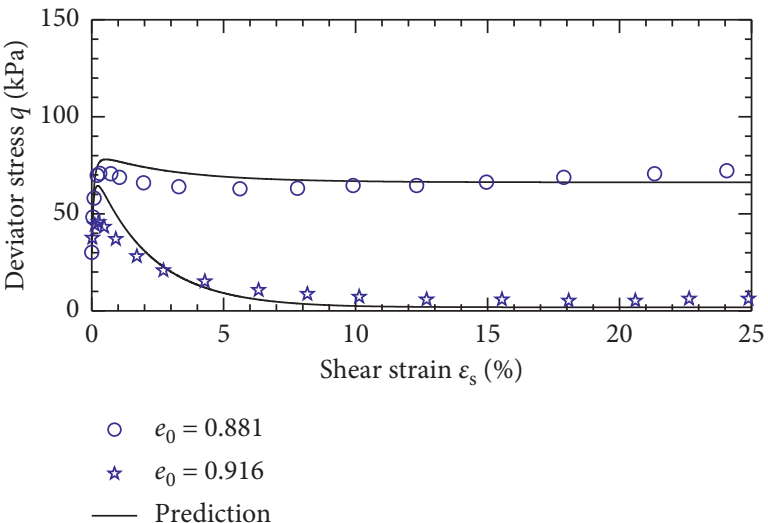

(a)

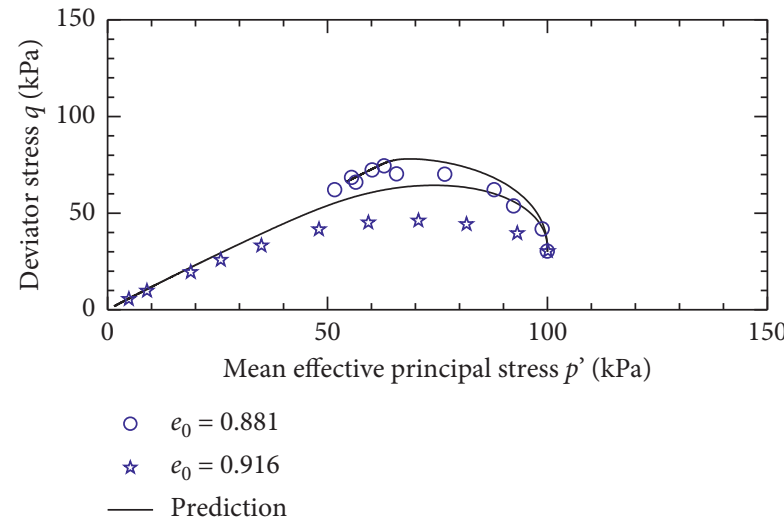

(b)

FIGURE 6: Simulations of the undrained behavior of anisotropically consolidated Firoozkuh sand.

\section{Conclusions}

This study presented an application of a stress-fractional model to simulate the constitutive behavior of granular soils, i.e., railroad ballast and Firoozkuh sand, under undrained or drained loads. Details of the model development and model implementation were provided. The main findings can be summarized as follows:

(1) The developed model contained twelve parameters, which can be identified through laboratory tests.

(2) Validation against the test results of crushed basalt revealed that the model can simulate the drained stress-strain response, including strain hardening, strain softening, volumetric dilation, and volumetric contraction, of railroad ballast. Small strain incremental loading can densify the material more quickly.

(3) Validation against the test results of Firoozkuh No. 161 sand showed that the model can simulate the undrained responses, e.g., liquefaction, partial liquefaction, and steady state, of sand with different initial material states and consolidation states.

\section{Data Availability}

The test data used to support the findings of this study are included within the article.

\section{Conflicts of Interest}

The authors declare that there are no conflicts of interest regarding the publication of this paper.

\section{Acknowledgments}

The financial support provided by the Scientific Research Project of Tianjin Education Commission (2019KJ145) is appreciated.

\section{References}

[1] S. Nimbalkar and B. Indraratna, "Improved performance of ballasted rail track using geosynthetics and rubber shockmat," Journal of Geotechnical and Geoenvironmental Engineering, vol. 142, no. 8, Article ID 04016031, 2016.

[2] A. Lashkari, A. Karimi, K. Fakharian, and F. KavianiHamedani, "Prediction of undrained behavior of isotropically 
and anisotropically consolidated Firoozkuh sand: instability and flow liquefaction," International Journal of Geomechanics, vol. 17, no. 10, Article ID 04017083, 2017.

[3] Y. Sun, Y. Gao, and Q. Zhu, "Fractional order plasticity modelling of state-dependent behaviour of granular soils without using plastic potential," International Journal of Plasticity, vol. 102, pp. 53-69, 2018.

[4] D. Lu, J. Liang, X. Du, C. Ma, and Z. Gao, "Fractional elastoplastic constitutive model for soils based on a novel 3D fractional plastic flow rule," Computers and Geotechnics, vol. 105, pp. 277-290, 2019.

[5] P.-F. Qu, Q.-Z. Zhu, and Y.-F. Sun, "Elastoplastic modelling of mechanical behavior of rocks with fractional-order plastic flow," International Journal of Mechanical Sciences, vol. 163, Article ID 105102, 2019.

[6] W. Sumelka, "Fractional viscoplasticity," Mechanics Research Communications, vol. 56, pp. 31-36, 2014.

[7] Y. Sun, W. Sumelka, Y. Gao, and S. Nimbalkar, "Phenomenological fractional stress-dilatancy model for granular soil and soil-structure interface under monotonic and cyclic loads," Acta Geotechnica, 2021.

[8] K. Hashiguchi and Z.-P. Chen, "Elastoplastic constitutive equation of soils with the subloading surface and the rotational hardening," International Journal for Numerical and Analytical Methods in Geomechanics, vol. 22, no. 3, pp. 197227, 1998.

[9] Y. Sun, Y. Gao, S. Song, and C. Chen, "Three-dimensional state-dependent fractional plasticity model for soils," International Journal of Geomechanics, vol. 20, no. 2, Article ID 04019161, 2020.

[10] Y. Sun, W. Sumelka, and Y. Gao, "Fractional plasticity for over-consolidated soft soil," Meccanica, 2021.

[11] A. Schofield and P. Wroth, Critical State Soil MechanicsMcGraw-Hill London, New York, NY, USA, 1968.

[12] B. O. Hardin and F. E. Richart, "Elastic wave velocities in granular soils," Journal of the Soil Mechanics and Foundations Division, vol. 89, no. 1, pp. 33-65, 1963.

[13] Y. Sun, Y. Gao, and C. Chen, "Critical-state fractional model and its numerical scheme for isotropic granular soil considering state-dependence," International Journal of Geomechanics, vol. 13, no. 9, Article ID 04018202, 2019.

[14] W. Salim and B. Indraratna, "A new elastoplastic constitutive model for coarse granular aggregates incorporating particle breakage," Canadian Geotechnical Journal, vol. 41, no. 4, pp. 657-671, 2004. 